Data Repository item 2004046

TABLE DR1: OXYGEN ISOTOPE RATIOS OF MINERAL SEPARATES, GEOCHRONOLOGY, AND SAMPLE LOCATIONS FOR PLUTONS OF THE GREAT BASIN

\begin{tabular}{|c|c|c|c|c|c|c|c|c|c|c|c|c|c|c|c|c|c|c|c|c|}
\hline 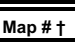 & Mountain Range & Specific Location & Sample & $\overline{\text { Age }}$ & " & $\begin{array}{ll}G^{80} \mathrm{O}(2 \mathrm{rc}) \\
\end{array}$ & $\begin{array}{l}\mathrm{G}^{8} \mathrm{O}(\mathrm{WR}) \S \\
\end{array}$ & ( & 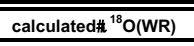 & Latitude & $\begin{array}{ll}\text { Longitude } \\
\end{array}$ & $\begin{array}{l}\text { Altitude } \\
\end{array}$ & $\overline{\mathrm{is \textrm {r }}}$ & 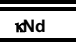 & $\begin{array}{ll}\mathrm{SiO}_{2} \\
\end{array}$ & $\overline{\mathrm{Al}_{2} \mathrm{O}_{3}}$ & CaOO & $\overline{\mathrm{N} \mathrm{Na}_{2} \mathrm{O}}$ & 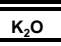 & $\begin{array}{l}\text { molecular A1203/(K2O+CaO+Na2O) } \\
\end{array}$ \\
\hline \multirow[t]{6}{*}{1} & Wasatch Range & Little Cottonwood stock & $\mathrm{LC}^{-15^{\ddagger}}$ & Tertiar, $28 \mathrm{Ma}$ & & $6.14 \pm 0.01$ & $8.8^{\mathrm{a}}$ & $5.21 \pm 0.07 \mathrm{Ttn}$ & $7.18-7.85$ & $40^{\circ} 32^{\prime}$ & $111^{1} 45^{\prime}$ & $2448 \mathrm{~m}$ & 0.70734 & -18.3 & 67.02 & 15.78 & 3.31 & 3.85 & 3.67 & 0.97 \\
\hline & & Little Cottonwood stock & ${\mathrm{LC}-2^{\ddagger}}^{\mathrm{a}}$ & Tertiary, $28 \mathrm{Ma}$ & & $5.47 \pm 0.47$ & & $4.02 \pm 0.06 \mathrm{Ttn}$ & & $40^{\circ} 32^{\prime}$ & $11^{1} 45^{\prime}$ & $1369 \mathrm{~m}$ & 0.70734 & -18.3 & 67.02 & 15.78 & 3.31 & 3.85 & 3.67 & 0.97 \\
\hline & & Little Cottonwood stock & LC- $-33^{ \pm}$ & Tertiar, $28 \mathrm{Ma}$ & & 5.96 & & $4.79 \pm 0.01 \mathrm{Ttn}$ & & $40^{\circ} 32^{\prime}$ & $11^{1} 45^{\prime}$ & $3170 \mathrm{~m}$ & 0.70734 & -18.3 & 67.02 & 15.78 & 3.31 & 3.85 & 3.67 & 0.97 \\
\hline & & Little Cottonwood stock & $\mathrm{LC}-34^{ \pm}$ & Tertiary, $28 \mathrm{Ma}$ & & 6.01 & & $4.78 \pm 0.01 \mathrm{Ttn}$ & & $40^{\circ} 32^{\prime}$ & $11^{1} 45^{\prime}$ & $3011 \mathrm{~m}$ & 0.70734 & -18.3 & 67.02 & 15.78 & 3.31 & 3.85 & 3.67 & 0.97 \\
\hline & & Little Cottonwood stock & LC. $5^{\ddagger}$ & Tertiary, $28 \mathrm{Ma}$ & & $5.82 \pm 0.01$ & & $4.24 \pm 0.04 \mathrm{Ttn}$ & & $40^{\circ} 32^{\prime}$ & $11^{1} 45^{\prime}$ & $2103 \mathrm{~m}$ & 0.70734 & -18.3 & 67.02 & 15.78 & 3.31 & 3.85 & 3.67 & 0.97 \\
\hline & & Little Cottonwood stock & LC- $9^{\ddagger}$ & Tertiary, $28 \mathrm{Ma}$ & & 6.01 & & $4.65 \pm 0.06 \mathrm{Ttn}$ & & $40^{\circ} 32^{\prime}$ & $11^{1} 45^{\prime}$ & $2890 \mathrm{~m}$ & 0.70734 & -18.3 & 67.02 & 15.78 & 3.31 & 3.85 & 3.67 & 0.97 \\
\hline 2 & Silver City Stock & & $99 \mathrm{~GB}-8$ & Tertiary, 32 Мa & 6.30 & & $0.6^{6}$ & & & $399^{\circ} 545^{\prime \prime}$ & $112^{\circ} 06^{6} 58^{\prime \prime}$ & & & & 60.6 & 15.1 & 4.2 & 3.7 & 3.7 & 0.85 \\
\hline 3 & Sheeprock Mountains & & 99GB-7 & Tertiary, $21 \mathrm{Ma}$ & $8.82 \pm 0.15$ & & & & & $39^{\circ} 50^{4} 2^{\prime \prime}$ & $112^{\circ} 24^{4} 3^{\prime \prime}$ & & 0.7064 & & 77 & 12.3 & 0.5 & 3.3 & 5 & 1.05 \\
\hline \multirow[t]{3}{*}{4} & Sheeprock Mountains & & $99 \mathrm{~GB}-4$ & Tertiary, 21 мa & $9.43 \pm 0.21$ & & & & & $399^{\circ} 555^{\prime \prime}$ & $112^{\circ} 30^{\prime 2} 25^{\prime \prime}$ & & 0.7064 & & 77 & 12.3 & 0.5 & 3.3 & 5 & 1.05 \\
\hline & & & $99 \mathrm{~GB}-5$ & Tertiary, 21 мa & $9.36 \pm 0.13$ & & & & & $399^{\circ} 545^{\prime \prime}$ & 112 $32^{\circ} 30^{\prime \prime 1}$ & & 0.7064 & & 77 & 12.3 & 0.5 & 3.3 & 5 & 1.05 \\
\hline & & & $99 \mathrm{~GB}-6$ & Tertiary, $21 \mathrm{Ma}$ & $9.61 \pm 0.14$ & & & & & $399^{\circ 5} 31 "$ & $112^{\circ} 28^{2} 29^{\prime \prime}$ & & 0.7064 & & 77 & 12.3 & 0.5 & 3.3 & 5 & 1.05 \\
\hline \multirow[t]{3}{*}{5} & Desert Mountain & & $99 \mathrm{~GB}-1$ & Tertiary, 27-29 Ma & $9.90 \pm 0.06$ & & $8.0^{\circ}$ & & & $39^{9} 46^{14} 2^{\prime \prime}$ & $112^{\circ} 35^{1} 10^{\prime \prime}$ & & & & 69.8 & 14.7 & 2.3 & 3.8 & 4.3 & 0.97 \\
\hline & & & $99 \mathrm{~GB}-2$ & Tertiary, 27-29 Ma & $9.32 \pm 0.09$ & & & & & $39^{9} 46^{\prime} 42^{\prime \prime}$ & $112^{\circ} 35^{1} 10^{\prime \prime}$ & & & & 69.8 & 14.7 & 2.3 & 3.8 & 4.3 & 0.97 \\
\hline & & & $99 \mathrm{~GB}-3$ & Tertiary, 27-29 Ma & $9.34 \pm 0.04$ & & & & & $39^{9} 46^{6} 42^{\prime \prime}$ & $112^{\circ} 35^{1} 10^{\prime \prime}$ & & & & 69.8 & 14.7 & 2.3 & 3.8 & 4.3 & 0.97 \\
\hline \multirow[t]{11}{*}{6} & House Range & Notch Peak & 95BR-006 & Jurassic, $169 \mathrm{Ma}$ & & $7.11 \pm 0.03$ & $9.3^{\mathrm{b}}$ & & & $39^{911}$ & $113^{\circ} 25^{\prime 3} 6^{\prime \prime}$ & & 0.70726 & -6.4 & 71.7 & 13.8 & 1.7 & 4 & 3.6 & 1.02 \\
\hline & & Notch Peak & 95BR-007 & Jurassic, $169 \mathrm{Ma}$ & & $7.21 \pm 0.07$ & & & & $39^{\circ} 11^{\prime}$ & $113^{\circ} 25^{\prime} 36^{\prime \prime}$ & & 0.70726 & -6.4 & 71.7 & 13.8 & 1.7 & 4 & 3.6 & 1.02 \\
\hline & & Notch Peak & 95BR-008 & Jurassic, $169 \mathrm{Ma}$ & & $7.38 \pm 0.06$ & & & & $39^{\circ} 11^{\prime}$ & $113^{\circ} 25^{\prime 3} 6^{\prime \prime}$ & & 0.70726 & -6.4 & 71.7 & 13.8 & 1.7 & 4 & 3.6 & 1.02 \\
\hline & & Notch Peak & 95BR-009 & Jurassic, $169 \mathrm{Ma}$ & & $7.45 \pm 0.02$ & & & & $39^{\circ} 10^{\prime} 40^{\prime \prime}$ & $113^{\circ} 25^{\prime 3} 6^{\prime \prime}$ & & 0.70726 & -6.4 & 71.7 & 13.8 & 1.7 & 4 & 3.6 & 1.02 \\
\hline & & Notch Peak & 95BR-010 & Jurassic, $169 \mathrm{Ma}$ & & $7.44 \pm 0.07$ & & & & $39^{\circ} 11^{\prime}$ & $113^{\circ} 25^{\prime 3} 6^{\prime \prime}$ & & 0.70726 & -6.4 & 71.7 & 13.8 & 1.7 & 4 & 3.6 & 1.02 \\
\hline & & Notch Peak & 95BR-012 & Jurassic, 169 Ma & & $7.33 \pm 0.00$ & & & & $39^{\circ} 11^{\prime}$ & $113^{\circ} 25^{\prime 3} 6^{\prime \prime}$ & & 0.70726 & -6.4 & 71.7 & 13.8 & 1.7 & 4 & 3.6 & 1.02 \\
\hline & & Notch Peak & 95BR-013 & Jurassic, $169 \mathrm{Ma}$ & & 7.53 & & & & $39^{\circ} 11^{\prime}$ & $113^{\circ} 25^{3} 6^{\prime \prime}$ & & 0.70726 & -6.4 & 71.7 & 13.8 & 1.7 & 4 & 3.6 & 1.02 \\
\hline & & Notch Peak & 95BR-014 & Jurassic, $169 \mathrm{Ma}$ & & $7.25 \pm 0.06$ & & & & $39^{\circ} 11^{102 "}$ & $113^{\circ} 25^{\prime 3} 6^{\prime \prime}$ & & 0.70726 & -6.4 & 71.7 & 13.8 & 1.7 & 4 & 3.6 & 1.02 \\
\hline & & Notch Peak & 95BR-015 & Jurassic, 169 Ma & & 7.42 & & & & $39^{911}$ & $113^{\circ} 25^{\prime 3} 6^{\prime \prime}$ & & 0.70726 & -6.4 & 71.7 & 13.8 & 1.7 & 4 & 3.6 & 1.02 \\
\hline & & Notch Peak & 95BR-016 & Jurassic, $169 \mathrm{Ma}$ & & $7.41 \pm 0.02$ & & & & $39^{\circ} 11^{\prime}$ & $113^{\circ} 25^{\prime 3} 6^{\prime \prime}$ & & 0.70726 & -6.4 & 71.7 & 13.8 & 1.7 & 4 & 3.6 & 1.02 \\
\hline & & Notch Peak & $N P-1^{\ddagger}$ & Jurassic, $169 \mathrm{Ma}$ & & $7.22 \pm 0.18$ & & $6.37 \pm 0.18 \mathrm{Ttn}$ & & $39^{911}$ & $113^{\circ} 25^{\prime}$ & & 0.70726 & -6.4 & 71.7 & 13.8 & 1.7 & 4 & 3.6 & 1.02 \\
\hline \multirow[t]{4}{*}{7} & Raft River Mountains & Clear Creek Canyon & 99GB-38 & Precambrian, 2,180 Ma & $8.90 \pm 0.05$ & & & & & 41957 $73^{\prime \prime}$ & $113^{\circ} 20^{\prime 3} 30^{\prime \prime}$ & & 0.764 & & & & & & & \\
\hline & & Clear Creek Canyon & 99GB-39 & Precambrian, 2,180 Ma & 8.72 & & & & & 41957 $708^{\prime \prime}$ & $113^{\circ} 21^{\circ} 4^{\prime \prime}$ & & 0.764 & & & & & & & \\
\hline & & Clear Creek Canyon & $996 \mathrm{~B}-40$ & Precambrian, 2,180 Ma & $8.08 \pm 0.09$ & & & & & 4195708" & $113^{\circ} 21^{104 "}$ & & 0.764 & & & & & & & \\
\hline & & Clear Creek Canyon & $996 \mathrm{~B}-41$ & Precambrian, 2,180 Ma & $8.74 \pm 0.02$ & & & & & $41^{\circ} 5720^{\prime \prime}$ & $113^{\circ} 18^{\prime 4} 0^{\prime \prime}$ & & 0.764 & & & & & & & \\
\hline 7 & Raft River Mountains & Dove Creek Pass & $996 \mathrm{~B}-45$ & Precambrian, 2, 2,80 Ma & $9.06 \pm 0.30$ & & & & & $41^{4} 4941^{\prime \prime}$ & 1113"3701" & & & & & & & & & \\
\hline \multirow[t]{4}{*}{8} & Grouse Creek Mountains & Imigrant Pass & $99 \mathrm{~GB}-34$ & Tertiary, 38 ма & $9.86 \pm 0.01$ & & $7.6^{\mathrm{b}}$ & $6.22 \mathrm{Tn}$ & & 41931'35" & $113^{\circ} 43^{2} 29^{\prime \prime}$ & & 0.709 & -17 & 70.4 & 14.2 & 2.1 & 3.9 & 3.5 & 1.01 \\
\hline & & Imigrant Pass & $996 \mathrm{~B}-35$ & Tertiary, $38 \mathrm{Ma}$ & $8.15 \pm 0.26$ & & & & & $41^{\circ} 31^{1} 40^{\prime \prime}$ & $113^{\circ} 43^{3} 36^{\prime \prime}$ & & 0.709 & -17 & 70.4 & 14.2 & 2.1 & 3.9 & 3.5 & 1.01 \\
\hline & & Imigrant Pass & 99GB-36 & Tertiary, $38 \mathrm{Ma}$ & $8.46 \pm 0.13$ & & & & & 41192300" & $113^{\circ} 45^{3} 33^{\prime \prime}$ & & 0.709 & -17 & 70.4 & 14.2 & 2.1 & 3.9 & 3.5 & 1.01 \\
\hline & & Imigrant Pass & $99 \mathrm{~GB}-37$ & Tertiary, 38 мa & $8.16 \pm 0.08$ & & & & & 41931'156" & $11^{3041} 11^{11}$ & & 0.709 & -17 & 70.4 & 14.2 & 2.1 & 3.9 & 3.5 & 1.01 \\
\hline \multirow[t]{2}{*}{8} & Grouse Creek Mountains & Ingham Pass & $996 B-46$ & Precambrian, 2,510 Ma & $7.99 \pm 0.06$ & & & & & 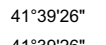 & 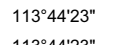 & & 0.714 & & & & & & & \\
\hline & Grouse Creek Mountains & $\begin{array}{l}\text { Ingham Pass } \\
\text { Ingham Pass, Red Butte granite }\end{array}$ & $\begin{array}{l}99 G \mathrm{~GB}-47 \\
99 \mathrm{~GB}-49\end{array}$ & $\begin{array}{l}\text { Precambrian, 2,510 Ma } \\
\text { Tertiary, } 25 \mathrm{Ma}\end{array}$ & $\begin{array}{l}8.36+0.81 \\
8.29 \pm 0.18\end{array}$ & & & & & $\begin{array}{l}41^{3} 3922^{2} 6^{\prime \prime} \\
41^{3} 399^{3} 0^{\prime \prime}\end{array}$ & $\begin{array}{l}113^{\circ} 44^{2} 23^{\prime \prime} \\
113^{\circ} 43^{3} 2^{\prime \prime}\end{array}$ & & $\begin{array}{c}0.714 \\
0.71559\end{array}$ & -26.3 & & & & & & \\
\hline & & & & & & & & & & & & & & & & & & & & \\
\hline \multirow[t]{6}{*}{9} & Pillot Range & McGinty monzograniti & 95BR-140 & Tertiary, $37 \mathrm{Ma}$ & & $5.98 \pm 0.01$ & $7.9^{\mathrm{b}}$ & & 7.07-8.12 & $41^{\circ} 11^{\prime}$ & $14^{\circ} 01^{1}$ & & 0.70717 & -13.3 & 70.3 & 14.7 & 2.4 & 3.9 & 4 & 0.97 \\
\hline & & McGinty monzogranitie & 95BR-141 & Tertiary, $37 \mathrm{Ma}$ & & 5.17 & & & & $41^{\circ} 11^{\prime}$ & $114^{\circ} 01^{\prime}$ & & 0.70717 & -13.3 & 70.3 & 14.7 & 2.4 & 3.9 & 4 & 0.97 \\
\hline & & McGinty monzogranite & 95BR-142 & Tertiary, $37 \mathrm{Ma}$ & & $6.15 \pm 0.04$ & & & & $41^{\circ} 11^{\prime}$ & $11^{\circ} 01^{1}$ & $1926 \mathrm{~m}$ & 0.70717 & -13.3 & 70.3 & 14.7 & 2.4 & 3.9 & 4 & 0.97 \\
\hline & & McGinty monzogranite & 95BR-143 & Tertiary, $37 \mathrm{Ma}$ & & 6.11 & & & & $41^{\circ} 11^{\prime}$ & $113^{\circ} 58^{5} 58^{\prime \prime}$ & & 0.70717 & -13.3 & 70.3 & 14.7 & 2.4 & 3.9 & 4 & 0.97 \\
\hline & & McGinty monzogranite & 95BR-144 & Tertiary, $37 \mathrm{Ma}$ & & $6.22 \pm 0.01$ & & & & $41^{\circ} 11^{\prime}$ & $114^{\circ} 01$ & & 0.70717 & -13.3 & 70.3 & 14.7 & 2.4 & 3.9 & 4 & 0.97 \\
\hline & & $\begin{array}{l}\text { McGinty monzogranite } \\
\text { McGinty monzocoranite }\end{array}$ & $\begin{array}{l}\text { 95BR-152 } \\
996 \mathrm{G}-31\end{array}$ & $\begin{array}{l}\text { Tertiary, } 37 \mathrm{Ma} \\
\text { Tertiary } 37 \mathrm{Ma}\end{array}$ & 92900.25 & $6.22 \pm 0.06$ & & & & $\begin{array}{l}41^{1911} \\
41^{11140^{\prime \prime}}\end{array}$ & $\begin{array}{l}113^{\circ} 58^{\prime} 58^{\prime \prime} \\
113^{\circ} 58^{\prime} 58^{\prime \prime}\end{array}$ & & $\begin{array}{l}0.70717 \\
0.70717\end{array}$ & $\begin{array}{l}-13.3 \\
-133 .\end{array}$ & $\begin{array}{l}70.3 \\
70.3\end{array}$ & $\begin{array}{l}14.7 \\
14.7\end{array}$ & $\begin{array}{l}2.4 \\
2.4\end{array}$ & $\begin{array}{l}3.9 \\
3.9\end{array}$ & $\begin{array}{l}4 \\
4\end{array}$ & $\begin{array}{l}0.97 \\
0.97\end{array}$ \\
\hline
\end{tabular}


Data Repository item 2004046

\begin{tabular}{|c|c|c|c|c|c|c|c|c|}
\hline \multirow[b]{3}{*}{9} & \multirow[b]{3}{*}{ Pilit Range } & \multirow{2}{*}{$\begin{array}{l}\text { McGinty monzogranite } \\
\text { McGinty mozzogranite }\end{array}$} & & Tertiary, $37 \mathrm{Ma}$ & $9.52 \pm 0.17$ & & \\
\hline & & & 99GB-33 & Tertiary, $37 \mathrm{Ma}$ & $9.42+0.15$ & & & \\
\hline & & Pilot Peak granitic dike & & & & $5.92 \pm 0.01$ & & \\
\hline \multirow{5}{*}{10} & & Pilot Peak granitic dike & 95BR-146 & Tertiary, 37 Ma & & $6.86 \pm 0.02$ & & \\
\hline & Silver Island Range & Crater Island South Stock & $99 \mathrm{~GB}-27$ & Jurassic $150-155 \mathrm{Ma}$ & $10.59 \pm 0.40$ & & & $5.90 \pm 0.15 \mathrm{Ttn}$ \\
\hline & & Crater Island South Stock & $99 \mathrm{~GB}-28$ & Jurassic $150-155 \mathrm{Ma}$ & $10.60 \pm 0.01$ & & & $5.83 \pm 0.01 \mathrm{Ttn}$ \\
\hline & & Crater Island South Stock & 99GB-29 & Jurassic 150-155 Ma & 10.36t0.06 & & & \\
\hline & & Crater Island South Stock & 99GB-30 & Jurassic $150-155 \mathrm{Ma}$ & 10.4550.16 & & & $5.922+0.00 \mathrm{Ttn}$ \\
\hline \multirow[t]{3}{*}{11} & Gold Hills & southern stock & 99GB-13 & Jurassic, $152 \mathrm{Ma}$ & 7.5600 .99 & & $9.0^{\mathrm{a}}$ & \\
\hline & & southern stock & 99GB-14 & Jurassic, $152 \mathrm{Ma}$ & $9.86 \pm 1.00$ & & & \\
\hline & & 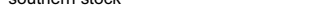 & 996B-15 & Jurassic, 152 Ma & 10.71 & & & \\
\hline \multirow[t]{3}{*}{12} & Deep Creek Range & Ipaban stock & 996B-10 & Tertiary, $39 \mathrm{Ma}$ & $10.43 \pm 0.08$ & & $9.6^{6}$ & \\
\hline & & Ipabah stock & 99GB-11 & Tertiary, $39 \mathrm{Ma}$ & $10.49 \pm 0.11$ & & & \\
\hline & & pabah stock & 99GB-12 & Tertiary, $39 \mathrm{Ma}$ & $8.10 \pm 0.95$ & & & \\
\hline \multirow[t]{13}{*}{13} & Beaver Dam Mountains & gneiss & 95BR-101 & Precambrian, 1379-1500 & & $7.94 \pm 0.05$ & & \\
\hline & & pegmatite & 95BR-102 & Precambrian, 1379-1500 & & $9.81 \pm 0.01$ & & \\
\hline & & pegmatite & 95BR-104 & Precambrian, 1379-1550 & & $8.13 \pm \pm 0.07$ & & \\
\hline & & pegmatte & 95BR-105 & Precambrian, 1379-1500 & & $6.44 \pm 0.01$ & & \\
\hline & & gneiss & 95BR-106 & Precambrian, 1379-1550 & & $6.31 \pm 0.01$ & & \\
\hline & & pegmatite & 95BR-108 & Precambrian, 1379-1500 & & $7.50 \pm 0.08$ & & \\
\hline & & pC basement gneiss & 95BR-109 & Precambrian, 1379-1500 & & $6.16 \pm 0.02$ & & \\
\hline & & gneiss & 95BR-110 & Precambrian, 1379-1500 & & $6.01 \pm 0.01$ & & \\
\hline & & pegmatite & 95BR-111 & Precambrian, 1379-1500 & & $6.94 \pm 0.07$ & & \\
\hline & & gneiss & 95BR-112 & Precambrian, 1379-1500 & & $5.62 \pm 0.10$ & & \\
\hline & & pegmatite & 95BR-113 & Precambrian, 1379-1500 & & $5.89 \pm 0.04$ & & \\
\hline & & pegmatite & 95BR-114 & Precambrian, 1379-1500 & & $6.07 \pm 0.03$ & & \\
\hline & & gneiss & 95BR-115 & Precambrian, 1379-1500 & & $6.55 \pm 0.01$ & & \\
\hline 14 & Kern Mountains & Tungstonia granite & 996B-16 & Cretaceous, $75 \mathrm{Ma}$ & 10.530.19 & & $9.6^{\mathrm{a}}$ & \\
\hline & & Tungstonia granite & 99GB-17 & Cretaceous, $75 \mathrm{Ma}$ & $10.53 \pm 0.02$ & & & \\
\hline 15a & Southern Snake Range & Lexington Creek & 99GB-20 & Cretaceous, $86 \mathrm{Ma}$ & $11.39 \pm 0.25$ & & $10.4,10.6^{\circ}$ & \\
\hline \multirow[t]{2}{*}{ 15b } & Southern Snake Range & Snake Creek Canyon & 99GB-18 & Jurassic, $160 \mathrm{Ma}$ & $11.83 \pm 0.02$ & & 10.2 to $12.2^{\mathrm{c}}$ & \\
\hline & & Snake Creek Canyon & 99GB-19 & Jurassic, $160 \mathrm{Ma}$ & $12.12+0.11$ & & & \\
\hline \multirow[t]{3}{*}{$15 \mathrm{c}$} & Southern Snake Range & Strawberry Creek/Osceola granodiorite & 99GB-21 & Jurassic, $160 \mathrm{Ma}$ & $11.56 \pm 0.08$ & & $9.6-13.2^{2^{\circ}}$ & \\
\hline & & Strawberry Creek/Osceola granodiorite & 99GB-22 & Jurassic, $160 \mathrm{Ma}$ & 10.64t0.25 & & & \\
\hline & & Strawberry Creek/Osceola granodiorite & 99GB-23 & Jurassic, $160 \mathrm{Ma}$ & $11.96 \pm 0.02$ & & & \\
\hline \multirow{4}{*}{16} & Goshute Mountains & White Horse granite & 95BR-133 & Jurassic, $157-160 \mathrm{Ma}$ & & $7.80 \pm 0.06$ & & \\
\hline & & White Horse pluton & 99GB-24 & Jurassic, $157-160 \mathrm{Ma}$ & 10.540 .12 & & $10.4^{b}$ & \\
\hline & & White Horse pluton & 99GB-25 & Jurassic, 157-160 Ma & 10.9550.11 & & & \\
\hline & & White Horse pluton & 99GB-26 & Jurassic, $157-160 \mathrm{Ma}$ & $10.49 \pm 0.12$ & & & \\
\hline \multirow[t]{4}{*}{$17 a$} & Toano Range & Silver Zone Pass & 996B-50 & Jurassic, $162 \mathrm{Ma}$ & 10.6850.12 & & $8.8^{a^{3}}$ & \\
\hline & & Silver Zone Pass & 996B-53 & Jurassic, 162 Ma & 10.86t0.32 & & & \\
\hline & Toano Range & Toana Springs granitie & 996B-51 & Cretaceous, $77 \mathrm{Ma}$ & $9.98 \pm 0.08$ & & & \\
\hline & & Toana Springs granite & 99GB-52 & Cretaceous, $77 \mathrm{Ma}$ & $10.12 \pm 0.30$ & & & \\
\hline \multirow[t]{4}{*}{18} & Dolly Varden Mountains & Merrose pluton & 95BR-135 & Jurassic, $165 \mathrm{Ma}$ & & $7.82 \pm 0.04$ & & \\
\hline & & Merrose pluton & 95BR-136 & Jurassic, $165 \mathrm{Ma}$ & & $6.24 \pm 0.02$ & & \\
\hline & & Melrose pluton & 99GB-54 & Jurassic, $165 \mathrm{Ma}$ & 10.420 .12 & & $9.1^{\mathrm{b}}$ & $5.89 \mathrm{Ttn}$ \\
\hline & & $\begin{array}{l}\text { Merloses pluton } \\
\text { Melrose pluton }\end{array}$ & $\begin{array}{l}999 \mathrm{~B}-55 \\
996 \mathrm{~B}-56\end{array}$ & $\begin{array}{l}\text { Jurarssic, } 165 \mathrm{Ma} \\
\text { Jurassic } 165 \mathrm{Ma}\end{array}$ & $\begin{array}{l}1.2550 .07 \\
10.72+0.13\end{array}$ & & & $6.40 \pm 0.13 \mathrm{Tn}$ \\
\hline
\end{tabular}

\begin{tabular}{|c|c|c|c|c|c|c|c|c|c|c|}
\hline $41^{\circ 1} 11^{\prime \prime}$ & $113^{\circ} 5858^{\prime \prime}$ & & 0.70017 & -13.3 & 70.3 & 14.7 & 2.4 & 3.9 & 4 & 0.97 \\
\hline $41^{\circ} 11^{1} 51^{\prime \prime}$ & $113^{\circ} 588^{2} 5^{\prime \prime}$ & & 0.70717 & -13.3 & 70.3 & 14.7 & 2.4 & 3.9 & 4 & 0.97 \\
\hline $41^{\circ} 11^{\prime}$ & $114^{\circ} 04^{\prime}$ & & & & & & & & & \\
\hline $\begin{array}{l}41^{\circ} 11^{\prime} \\
\end{array}$ & $\begin{array}{l}114^{\circ} 04^{\prime} \\
\end{array}$ & & & & & & & & 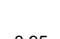 & 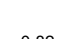 \\
\hline $41^{\circ} 01^{105 "}$ & $113^{\circ} 46^{6} \cdot 4^{\prime \prime}$ & & 0.7062 & -4.9 & 63.5 & 15 & 4.33 & 3.78 & 3.95 & 0.82 \\
\hline $41^{\circ} 01^{1} 0^{\prime \prime}$ & $113^{\circ} 466^{6} 4^{\prime \prime}$ & & 0.7062 & -4.9 & 63.5 & 15 & 4.33 & 3.78 & 3.95 & 0.82 \\
\hline $41^{\circ} 02^{2} 24^{\prime \prime}$ & 111384731" & & 0.7062 & -4.9 & 63.5 & 15 & 4.33 & 3.78 & 3.95 & $\begin{array}{l}0.82 \\
\end{array}$ \\
\hline $41^{\circ} 00^{3} 0^{11}$ & $113^{\circ} 45^{3} 37^{\prime \prime}$ & & 0.7062 & -4.9 & 63.5 & 15 & 4.33 & 3.78 & 3.95 & 0.82 \\
\hline $40^{\circ} 05^{2} 29^{\prime \prime}$ & $113^{\circ} 455^{57}$ & & 0.7097 & -8.6 & 73.95 & 13.35 & 1.3 & 2.88 & 5.4 & 1.03 \\
\hline $40^{\circ} 05^{2} 28^{\prime \prime}$ & $11^{1046^{\circ} 011^{11}}$ & & 0.7097 & -8.6 & 73.95 & 13.35 & 1.3 & 2.88 & 5.4 & 1.03 \\
\hline $40^{\circ} 0755^{\prime \prime}$ & $113^{\circ} 455^{35}{ }^{\prime}$ & & 0.7097 & -8.6 & 73.95 & 13.35 & 1.3 & 2.88 & 5.4 & 1.03 \\
\hline $39^{\circ} 47444^{\prime \prime}$ & $113^{\circ} 500^{2} 4^{\prime \prime}$ & & 0.71659 & -16.8 & 71.2 & 14.2 & 2.1 & 3.4 & 4.4 & 1.00 \\
\hline $\begin{array}{l}39{ }^{\circ} 50^{\circ} 43^{\prime \prime} \\
\end{array}$ & $113^{\circ} 4854^{\prime \prime}$ & & 0.71659 & -16.8 & 71.2 & 14.2 & 2.1 & 3.4 & 4.4 & 1.00 \\
\hline 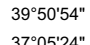 & 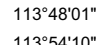 & & 0.71659 & -16.8 & 71.2 & 14.2 & 2.1 & 3.4 & 4.4 & 1.00 \\
\hline $37^{\circ} 05^{2} 24^{\prime \prime}$ & $113^{\circ} 54^{4} 10^{\prime \prime}$ & $1250 \mathrm{~m}$ & & & & & & & & \\
\hline $\begin{array}{l}37^{\circ} 05^{\prime} \\
37^{\circ}\end{array}$ & 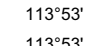 & $\begin{array}{l}1250 \mathrm{~m} \\
11316\end{array}$ & & & & & & & & \\
\hline $\begin{array}{l}37^{\circ} 05^{\prime} \\
\end{array}$ & $\begin{array}{l}11^{\circ} 53^{\prime} \\
1\end{array}$ & $1316 \mathrm{~m}$ & & & & & & & & \\
\hline $\begin{array}{ll}37^{\circ} 05^{4} 46^{\prime \prime} \\
37^{\circ}\end{array}$ & 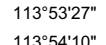 & $\begin{array}{l}1442 \mathrm{~m} \\
1341\end{array}$ & & & & & & & & \\
\hline $\begin{array}{l}37^{0} 05^{\prime} \\
37^{2} 05^{2}\end{array}$ & $\begin{array}{ll}13^{\circ} 54^{4} 10^{10} \\
1\end{array}$ & $1341 \mathrm{~m}$ & & & & & & & & \\
\hline $\begin{array}{l}37^{3} 05^{\prime} \\
277^{2}\end{array}$ & 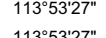 & $1341 \mathrm{~m}$ & & & & & & & & \\
\hline $\begin{array}{l}3705^{3} \\
377^{2}\end{array}$ & 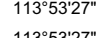 & $1366 \mathrm{~m}$ & & & & & & & & \\
\hline $\begin{array}{l}3705^{3} \\
377^{2}=5^{2}\end{array}$ & 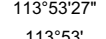 & $\begin{array}{l}1308 \mathrm{~m} \\
1371\end{array}$ & & & & & & & & \\
\hline $\begin{array}{l}37^{0} 05^{\prime} \\
7000\end{array}$ & 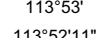 & $1371 \mathrm{~m}$ & & & & & & & & \\
\hline $\begin{array}{ll}37^{0} 05^{5} 59^{\prime \prime} \\
37^{\prime \prime}\end{array}$ & 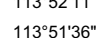 & $\begin{array}{l}1381 \mathrm{~m} \\
1343\end{array}$ & & & & & & & & \\
\hline $\begin{array}{l}37^{3} 05^{\prime} \\
377^{2}\end{array}$ & 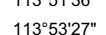 & $\begin{array}{l}1343 \mathrm{~m} \\
1369 \mathrm{~m}\end{array}$ & & & & & & & & \\
\hline $37^{\circ} 06^{\prime} 13$ & $11_{13}^{\circ} 53^{2} 27^{n}$ & $\begin{array}{l}1360 \mathrm{~m} \\
1396 \mathrm{~m}\end{array}$ & & & & & & & & \\
\hline $39^{\circ} 44^{\circ} 4^{\prime \prime}$ & $11^{10^{\circ} 13^{1} 9^{\prime \prime}}$ & & 0.72114 & -19.1 & 73.62 & 14.26 & 1.93 & 3.51 & 3.15 & 1.12 \\
\hline $39^{3944} 4^{6 "}$ & $11^{4} 11^{\circ 2} 29^{\prime \prime}$ & & 0.72114 & -19.1 & 73.62 & 14.26 & 1.93 & 3.51 & 3.15 & 1.12 \\
\hline $38^{\circ} 50^{\circ} 45^{\prime \prime}$ & $114^{\circ} 11118^{\prime \prime}$ & & 0.7114 & -12.9 & 71.8 & 15.3 & 1.8 & 3.3 & 3.9 & 1.18 \\
\hline $39^{\circ} 55^{3} 31 "$ & $11^{\circ} 44^{411}$ & & 0.7071 & & 71.425 & 15.175 & 2.27 & 3.25 & 3.45 & 1.15 \\
\hline $38^{\circ} 55^{2} 22^{\prime \prime}$ & $114^{\circ} 1358^{\prime \prime}$ & & 0.7071 & & 71.425 & 15.175 & 2.27 & 3.25 & 3.45 & 1.15 \\
\hline $39^{\circ} 04^{\circ} 02^{\prime \prime}$ & $11^{\circ} 15^{1} 0^{\circ "}$ & & 0.7075 & & & & & & & \\
\hline $39^{\circ} 04^{4} 02^{\prime \prime}$ & $114^{\circ} 15^{10} 0^{\prime \prime}$ & & 0.7075 & & & & & & & \\
\hline 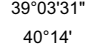 & 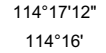 & & $\begin{array}{l}0.775 \\
0.7072\end{array}$ & -5.5 & 71.5 & 14.2 & 2.32 & 3.62 & 3.94 & 0.98 \\
\hline $40^{\circ} 11^{4} 49^{\prime \prime}$ & $1141^{\circ} 10^{\circ} 9^{\prime \prime}$ & & 0.7072 & -5.5 & 71.5 & 14.2 & 2.32 & 3.62 & 3.94 & 0.98 \\
\hline $40^{\circ} 11^{4} 49^{\prime \prime}$ & $114^{\circ} 18^{\circ} 0^{\prime \prime}$ & & 0.7072 & -5.5 & 71.5 & 14.2 & 2.32 & 3.62 & 3.94 & 0.98 \\
\hline $40^{\circ} 11^{4} 45^{\prime \prime}$ & $114^{\circ} 18^{2} 26^{\prime \prime}$ & & 0.7072 & -5.5 & 71.5 & 14.2 & 2.32 & 3.62 & 3.94 & 0.98 \\
\hline $40^{\circ} 54^{4} 30^{\prime \prime}$ & $114^{\circ} 18^{2} 26^{\prime \prime}$ & & 0.7052 & -2.5 & 68.6 & 15.3 & 3.19 & 3.53 & 3.14 & 1.02 \\
\hline $40^{\circ} 54^{4} 47^{\prime \prime}$ & $114^{\circ} 1758^{\prime \prime}$ & & 0.7052 & -2.5 & 68.6 & 15.3 & 3.19 & 3.53 & 3.14 & 1.02 \\
\hline $40^{\circ} 59^{\prime 17}$ & $114^{\circ} 1911^{\prime \prime}$ & & 0.725 & -23.2 & 72.85 & 15.38 & 1.04 & 4.07 & 4.17 & 1.17 \\
\hline $40^{\circ} 59^{\prime} 33^{\prime \prime}$ & $114^{\circ} 19211^{11}$ & & 0.725 & -23.2 & 72.85 & 15.38 & 1.04 & 4.07 & 4.17 & 1.17 \\
\hline $40^{\circ} 20^{\prime}$ & $114^{\circ} 344^{2} 7^{\prime \prime}$ & $2070 \mathrm{~m}$ & 0.7067 & -5.4 & 58.7 & 16.7 & 4.36 & 4.39 & 5.78 & 0.78 \\
\hline $40^{\circ} 20^{\circ}$ & $114^{\circ} 3427^{\prime \prime}$ & $2035 \mathrm{~m}$ & 0.7067 & -5.4 & 58.7 & 16.7 & 4.36 & 4.39 & 5.78 & 0.78 \\
\hline $40^{\circ} 20^{\prime 19 "}$ & $114^{\circ} 344^{27 "}$ & & 0.7067 & -5.4 & 58.7 & 16.7 & 4.36 & 4.39 & 5.78 & 0.78 \\
\hline $40^{\circ} 20^{\circ 19} 9^{\prime \prime}$ & $114^{\circ} 3450^{\prime \prime}$ & & 0.7067 & -5.4 & 58.7 & 16.7 & 4.36 & 4.39 & 5.78 & 78 \\
\hline $40^{\circ} 21^{12} 26^{\prime \prime}$ & $114^{4} 354^{41}$ & & 0.7067 & -5.4 & 58.7 & 16.7 & 4.36 & 4.39 & 5.78 & 0.78 \\
\hline
\end{tabular}


Data Repository item 2004046

\begin{tabular}{|c|c|c|c|c|c|c|c|c|}
\hline \multirow[t]{6}{*}{19} & \multirow{6}{*}{ Mormon Mountains } & East Mormom Mountains gneiss & & \multicolumn{2}{|l|}{ Precambrian, $1500 \mathrm{Ma}$} & \multicolumn{3}{|l|}{$6.01 \pm 0.02$} \\
\hline & & East Mormom Mountains sneiss & $968 B-006$ & Precambrian, $1500 \mathrm{Ma}$ & & $6.47+0.06$ & & \\
\hline & & East Mormom Mountains gneiss & & Precambrian, $1500 \mathrm{Ma}$ & & & & \\
\hline & & central Mormon Mountains gneiss & 96BR-010 & Precambrian, $1500 \mathrm{Ma}$ & & $5.62+0.12$ & & \\
\hline & & central Mormon Mountains gneiss & & Precambrian, $1500 \mathrm{Ma}$ & & $6.06 \pm 0.02$ & & \\
\hline & & West Mormon Mountains gneiss & $96 \mathrm{BR}-016$ & Precambrian, $1500 \mathrm{Ma}$ & & $4.92 \pm 0.01$ & & \\
\hline 20 & \multirow{3}{*}{$\begin{array}{l}\text { Antelope Range } \\
\text { Delcer Buttes }\end{array}$} & Kingsley granite & & Tertiary, $33 \mathrm{Ma}$ & & $6.78 \pm 0.04$ & & \\
\hline 22 & & & $996 \mathrm{~B}-59$ & Jurassic & $10.76 \pm 0.17$ & & & \\
\hline \multirow{4}{*}{23} & & & $99 \mathrm{~GB}-60$ & Jurassic & $10.43 \pm 0.03$ & & & \\
\hline & \multirow{3}{*}{ Cherry Creek Mountains } & $\begin{array}{l}\text { Cherry Y reeke graninte } \\
\text { Cherr Creek granite }\end{array}$ & $\begin{array}{l}95 B R-130 \\
996-62\end{array}$ & $\begin{array}{l}\text { Tetriary, } 32 \mathrm{Ma} \\
\text { Tetriag }\end{array}$ & $881+021$ & 7.06 & $5.8^{\mathrm{a}}$ & \\
\hline & & $\begin{array}{l}\text { Cherry Creee graninte } \\
\text { Chery Creek granite }\end{array}$ & $\begin{array}{l}99 \mathrm{~GB}-\mathrm{B} 2 \\
99 \mathrm{~B}-63\end{array}$ & $\begin{array}{l}\text { Tetriara, } 32 \mathrm{Ma} \\
\text { Tetriar, } 32 \mathrm{Ma}\end{array}$ & $\begin{array}{l}8.8110 .21 \\
4.8440 .96\end{array}$ & & & \\
\hline & & Cherry Creek granite & $996 \mathrm{~B}-64$ & Tertiary, $32 \mathrm{Ma}$ & 9.19 & & & \\
\hline 24 & Egan Range & Gold Creek granite & 95BR-131 & Tertiary & & 6.78 & & \\
\hline & Egan Range & Warm Springs granite & $996 \mathrm{~B}-65$ & Tertiary & $-7.63 \pm 0.16$ & & & $-13.45 \mathrm{Bt}$ \\
\hline \multirow{12}{*}{$\begin{array}{l}25 a \\
25 b\end{array}$} & Ruby Mountains & Little Bald Mountain & $99 \mathrm{~GB}-66$ & Tertiary & $12.09 \pm 0.07$ & & & \\
\hline & \multirow{11}{*}{ Ruby Mountains } & Harrison Pass & 99GB-71 & Tertiary, 35-37 Ma & $11.13 \pm 0.04$ & & $9.5^{\mathrm{a}}$ & \\
\hline & & Harrison Pass & $99 \mathrm{~GB}-72$ & Tertiary, 35-37 Ma & $9.07 \pm 0.19$ & & & \\
\hline & & Harrison Pass & $99 \mathrm{~GB}-73$ & Tertiary, 35-37 Ma & $6.52 \pm 0.11$ & & & \\
\hline & & Harrison Pass & $99 \mathrm{~GB}-74$ & Tertiary, 35-37 Ma & $10.58+0.04$ & & & $6.87 \pm 0.54 \mathrm{Gnt}$ \\
\hline & & Harrison Pass & $99 G \mathrm{~B}-75$ & Tertiary, 35-37 Ma & $\begin{array}{l}10.3000 \pm 0.04 \\
10.860 .06\end{array}$ & & & $0.08 \mathrm{ta} .04 \mathrm{Gm}$ \\
\hline & & Harrison Pass & $99 \mathrm{~GB}-76$ & Tertiary, 35-37 Ma & $10.42+0.12$ & & & \\
\hline & & Harrison Pass & 99GB-77 & Tertiary, $35-37 \mathrm{Ma}$ & $10.16 \pm 0.25$ & & & \\
\hline & & Harrison Pass & 99GB-78 & Terriary, 35-37 Ma & $10.79 \pm 0.04$ & & & \\
\hline & & Harrison Pass & 99GB-79 & Tertiary, 35-37 Ma & $10.69 \pm 0.08$ & & & \\
\hline & & Harrison Pass & RM-6 & Tertiary, 35-37 Ma & & $7.68 \pm 0.06$ & & \\
\hline & & Harrison Pass & RM-8 & Tertiary, 35-37 Ma & & $5.88 \pm 0.05$ & & \\
\hline \multirow{2}{*}{$25 b$} & \multirow[t]{2}{*}{ Ruby Mountains } & McCutcheon Creek & $99 \mathrm{BB}-80$ & Jurassic, $160 \mathrm{Ma}$ & $10.51 \pm 0.06$ & & & \\
\hline & & McCutcheon Creek & $99 \mathrm{~GB}-82$ & Jurassic, $160 \mathrm{Ma}$ & $10.07 \pm 0.25$ & & & \\
\hline \multirow{2}{*}{$25 c$} & \multirow{2}{*}{ Ruby Mountains } & Lamoille Canyon & RM-15 & Tertiary, $29 \mathrm{Ma}$ & & 8.05 & & \\
\hline & & Lamoille Canyon & RM-4 & Cretaceous, $80 \mathrm{Ma}$ & & 9.89 & & \\
\hline \multirow{6}{*}{26} & \multirow[t]{6}{*}{ East Humboldt Range } & Rattlesnake Canyon & RM-13 & Tertiary, $38 \mathrm{Ma}$ & & $5.42 \pm 0.29$ & & \\
\hline & & & RM-18 & Cretaceous, $73 \mathrm{Ma}$ & & $7.76 \pm 0.02$ & & \\
\hline & & & RM-19 & Tertiary, $40 \mathrm{Ma}$ & & $5.76 \pm 0.06$ & & \\
\hline & & & RM-20 & Tertiary, $29 \mathrm{Ma}$ & & $5.34 \pm 0.08$ & & $970+0.04 \mathrm{Gnt}$ \\
\hline & & Angellake orthogneiss & $\begin{array}{l}\mathrm{R}-2 \mathrm{-24} \\
\mathrm{RM}-5\end{array}$ & $\begin{array}{l}\text { Tertary, } \\
\text { Tertiar. } 29 \mathrm{Ma}\end{array}$ & & & & $9.70 \pm 0.04 \mathrm{Gnt}$ \\
\hline & & $\begin{array}{l}\text { Angen Lace ornognelss } \\
\text { Horse Creek }\end{array}$ & $\mathrm{RM}-7$ & Tertiary, $35 \mathrm{Ma}$ & & $\begin{array}{l}5.360 \\
6.28 \pm 0.02\end{array}$ & & \\
\hline 27 & \multirow{2}{*}{ Cortez Mountains } & Cottonwood Canyon & $99 G \mathrm{~B}-67$ & Cretaceous & $11.51 \pm 0.08$ & & $9.9^{b}$ & \\
\hline & & Cottonwood Canyon & $99 \mathrm{~GB}-68$ & Cretaceous & $11.73 \pm 0.06$ & & & \\
\hline 28 & Dry Hills & & $99 \mathrm{~GB}-70$ & Cretaceous & 11.49 & & & \\
\hline 29 & White Pine Range & & 97BR-101 & Tertiary & & 8.07 & $10.8^{b}$ & \\
\hline & & & 97BR-102 & Tertiary & & 7.76 & & \\
\hline & & & 97BR-103 & Tertiary & & $8.53 \pm 0.01$ & & \\
\hline 30 & Grant Range & Troy granite & 96BR-018 & Cretaceous, $87 \mathrm{Ma}$ & & 7.79 & $11.1^{b}$ & \\
\hline & & & 96BR-019 & Cretaceous, $87 \mathrm{Ma}$ & & 8.18 & & \\
\hline & & & 97BR-105 & Cretaceous, $87 \mathrm{Ma}$ & & 8.05 & & \\
\hline & & & 97BR-106 & Cretaceous, $87 \mathrm{Ma}$ & & $8.34 \pm 0.01$ & & \\
\hline & & & 97BR-107 & Cretaceous, $87 \mathrm{Ma}$ & & $7.94 \pm 0.01$ & & \\
\hline & & & 97BR-108 & Cretaceous, $87 \mathrm{Ma}$ & & $8.10 \pm 0.06$ & & \\
\hline 31 & Northern Quinn Range & lower Willow Creek & 96BR-017 & Tertiary, $27 \mathrm{Ma}$ & & $7.89 \pm 0.01$ & & \\
\hline 32 & Buffalo Mountain & & $996 \mathrm{~B}-83$ & Jurassic, $153 \mathrm{Ma}$ & 10.71 & & $9.3^{\mathrm{a}}$ & \\
\hline 33 & Edna Mountain & & 99GB-86 & Cretaceous, 88-92 Ma & $10.44 \pm 0.00$ & & $9.9^{\mathrm{b}}$ & \\
\hline 34 & Osgood Mountains & & $99 \mathrm{~GB}-87$ & Cretaceous $90 \mathrm{Ma}$ & $1142+0.43$ & & $9.9^{a}$ & $689+0.11$ th \\
\hline
\end{tabular}

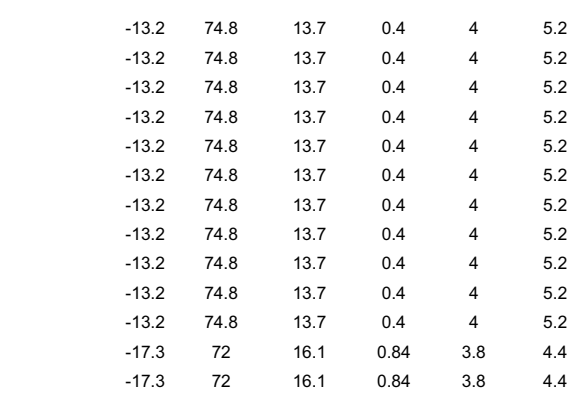


Data Repository item 2004046

\begin{tabular}{|c|c|c|c|c|c|c|c|c|c|c|c|c|c|c|c|c|c|c|c|c|}
\hline \multirow{3}{*}{35} & & & 99GB-88 & Cretaceous, $90 \mathrm{Ma}$ & $11.82 \pm 0.07$ & & & & & $41^{\circ} 07^{\prime}$ & $117^{\circ} 15^{\prime} 30^{\prime \prime}$ & & 0.7058 & -3.4 & 65.9 & 16.8 & 4.3 & 3.6 & 2.8 & 1.00 \\
\hline & \multirow{2}{*}{\multicolumn{2}{|c|}{ Slumbering Hills }} & 996B-89 & $\begin{array}{l}\text { Cretaceous, } 90 \text { Ma } \\
\text { Cretatcoos }\end{array}$ & $\begin{array}{l}12.78+0.27 \\
9.57+0.03\end{array}$ & & & & & 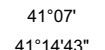 & 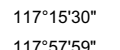 & & 0.7058 & -3.4 & 65.9 & 16.8 & 4.3 & 3.6 & 2.8 & 1.00 \\
\hline & & & 的B -92 & $\begin{array}{l}\text { Cretacoeus } \\
\text { Cretaceous }\end{array}$ & $\begin{array}{l}9.5 .7 \pm 0.022 \\
9.34 \pm .09\end{array}$ & & & & & $\begin{array}{l}41 / 143^{\prime} \\
41^{1} 143^{\prime \prime}\end{array}$ & $117^{\circ} 57^{\prime \prime 59^{\prime \prime}}$ & & & & & & & & & \\
\hline 36 & Eugene Mountains & & 99GB-94 & Cretaceous & $\begin{array}{l}.0 .01 .0 .000 \\
11.0303\end{array}$ & & $10.2^{\mathrm{b}}$ & & & $40^{4} 41^{\prime 2} 23^{\prime \prime}$ & $118^{\circ} 1457^{\prime \prime}$ & & & & 67 & 15.1 & 3.3 & 3.6 & 3.5 & 0.96 \\
\hline \multirow[t]{5}{*}{$37 a$} & Toiyabe Range & Austin pluton & 97TY-002 & Jurassic, $142 \mathrm{Ma}$ & & 8.58 & $10.4^{\mathrm{a}}$ & & $10.14-10.34$ & & $117^{\circ} 04^{4} 58^{\prime \prime}$ & $1900 \mathrm{~m}$ & 0.7068 & -4.2 & 67.2 & 14.9 & 3.3 & 3.6 & 3.8 & 0.93 \\
\hline & & Austin pluton & 97TY-004 & Jurassic, $142 \mathrm{Ma}$ & & $8.54 \pm 0.07$ & & & & & $117^{\circ} 02555^{\prime \prime}$ & $2150 \mathrm{~m}$ & 0.7068 & -4.2 & 67.2 & 14.9 & 3.3 & 3.6 & 3.8 & 0.93 \\
\hline & & Austin pluton & 97TY-005 & Jurassic, $142 \mathrm{Ma}$ & & $8.42+0.01$ & & & & & $117^{\circ} 02555^{\prime \prime}$ & $2250 \mathrm{~m}$ & 0.7068 & -4.2 & 67.2 & 14.9 & 3.3 & 3.6 & 3.8 & 0.93 \\
\hline & & Austin pluton & 97TY-006 & Jurassic, $142 \mathrm{Ma}$ & & 8.5220 .03 & & & & & $117^{\circ} 02^{\prime} 14^{\prime \prime}$ & $2300 \mathrm{~m}$ & 0.7068 & -4.2 & 67.2 & 14.9 & 3.3 & 3.6 & 3.8 & 0.93 \\
\hline & & Austin pluton & 97TY-007 & Jurassic, $142 \mathrm{Ma}$ & & 8.5770 .02 & & & & & $117^{\circ} 03^{3} 20^{\prime \prime}$ & $2240 \mathrm{~m}$ & 0.7068 & -4.2 & 67.2 & 14.9 & 3.3 & 3.6 & 3.8 & 0.93 \\
\hline & & Austin pluton & 97тY-008 & Jurassic, $142 \mathrm{Ma}$ & & $8.622 \pm 0.05$ & & & & & $117^{\circ} 03^{3} 20^{\prime \prime}$ & & 0.7068 & -4.2 & 67.2 & 14.9 & 3.3 & 3.6 & 3.8 & 0.93 \\
\hline & & Austin pluton & $97 \mathrm{TY}-010$ & Jurassic, $142 \mathrm{Ma}$ & & 8.5770 .14 & & & & & $11^{\circ} 58^{\prime}$ & & 0.7068 & -4.2 & 67.2 & 14.9 & 3.3 & 3.6 & 3.8 & 0.93 \\
\hline & & Austin pluton & $97 T \mathrm{~T}-012 \mathrm{~A}$ & Jurassic, $142 \mathrm{Ma}$ & & 8.5550 .03 & & & & & $116^{\circ} 57^{\prime} 14 "$ & & 0.7068 & -4.2 & 67.2 & 14.9 & 3.3 & 3.6 & 3.8 & 0.93 \\
\hline & & Austin pluton & $97 T \mathrm{~T}-012 \mathrm{~B}$ & Jurassic, $142 \mathrm{Ma}$ & & 8.5770 .03 & & & & & $116^{\circ} 57^{\prime} 14^{\prime \prime}$ & & 0.7068 & -4.2 & 67.2 & 14.9 & 3.3 & 3.6 & 3.8 & 0.93 \\
\hline & & Austin pluton & 99GB-128 & Jurassic, $142 \mathrm{Ma}$ & 11.84 & & & & & $39^{\circ} 2925^{\prime \prime}$ & 117"03'24" & & 0.7068 & -4.2 & 67.2 & 14.9 & 3.3 & 3.6 & 3.8 & 0.93 \\
\hline & & Austin pluton & 99GB-129 & Jurassic, $142 \mathrm{Ma}$ & $11.46 \pm 0.11$ & & & & & $39^{\circ} 288^{4} 2^{n}$ & $117^{\circ} 01^{1} 45^{\prime \prime}$ & & 0.7068 & -4.2 & 67.2 & 14.9 & 3.3 & 3.6 & 3.8 & 0.93 \\
\hline & & Austin pluton & 99GB-130 & Jurassic, $142 \mathrm{Ma}$ & $11.67 \pm 0.08$ & & & $7.18 \pm 0.16 \mathrm{Ttn}$ & & $39^{\circ} 25511 "$ & $116^{\circ} 58^{\prime 1} 11 "$ & & 0.7068 & -4.2 & 67.2 & 14.9 & 3.3 & 3.6 & 3.8 & 0.93 \\
\hline & & Austin pluton & $996 \mathrm{G}-131$ & Jurassic, $142 \mathrm{Ma}$ & $11.74 \pm 0.26$ & & & & & $39^{\circ} 25511 "$ & $116^{\circ} 58^{\prime 11} 1 "$ & & 0.7068 & -4.2 & 67.2 & 14.9 & 3.3 & 3.6 & 3.8 & 0.93 \\
\hline \multirow[t]{8}{*}{$37 \mathrm{~b}$} & Toiyabe Range & Birch Creek pluton & $97 \mathrm{TY}-013$ & Cretaceous, $75 \mathrm{Ma}$ & & 9.96 & & & & $39^{\circ} 2350^{\prime \prime}$ & $117^{\circ} 02^{2} \cdot 11$ & & 0.70766 & -6.4 & & & & & & \\
\hline & & Birch Creek pluton & 97TY-014 & Cretaceous, $75 \mathrm{Ma}$ & & $9.63 \pm 0.02$ & & & & $39^{\circ 23} 34^{\prime \prime}$ & $17^{\circ} 01^{152}$ & & 0.70766 & -6.4 & & & & & & \\
\hline & & Birch Creek pluton & 97TY-016 & Cretaceous, $75 \mathrm{Ma}$ & & 9.52 & & & & $39^{\circ 2257 "}$ & $117^{\circ} 2^{\prime}$ & & 0.70766 & -6.4 & & & & & & \\
\hline & & Birch Creek pluton & $97 T \mathrm{Y}-017$ & Cretaceous, $75 \mathrm{Ma}$ & & $9.44 \pm 0.03$ & & & & $39^{\circ 2250^{\prime \prime}}$ & $117^{\circ} 00^{\circ} 58^{\prime \prime}$ & & 0.70766 & -6.4 & & & & & & \\
\hline & & Birch Creek pluton & 996B-132 & Cretaceous, $75 \mathrm{Ma}$ & $13.05 \pm 0.14$ & & & & & $39^{\circ 22228^{n}}$ & $117^{\circ} 00^{\prime 19} 19^{\prime \prime}$ & & 0.70766 & -6.4 & & & & & & \\
\hline & & $\begin{array}{l}\text { Birch Creek pluton } \\
\text { Birch Creak }\end{array}$ & 996B-133 & $\begin{array}{l}\text { Cretaceous, } 75 \mathrm{Ma} \\
\text { Cretaceos, } 755\end{array}$ & $13.03 \pm 0.10$ & & & & & 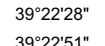 & $117^{\circ} 00^{\prime 19 " 19}$ & & 0.70766 & -6.4 & & & & & & \\
\hline & & $\begin{array}{l}\text { Birch Creek pluton } \\
\text { Birch Creek pluton }\end{array}$ & $\begin{array}{l}99 G B-134 \\
996 B-135\end{array}$ & $\begin{array}{l}\text { Cretaceous, } 75 \mathrm{Ma} \\
\text { Cretaceous, } 75 \mathrm{Ma}\end{array}$ & $\begin{array}{l}13.23 \pm 0.27 \\
12.96 \pm 0.10\end{array}$ & & & & & 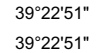 & $\begin{array}{l}117^{\circ} 01^{10} 1 " 1 \\
117^{\circ} 010^{\prime \prime} 11\end{array}$ & & $\begin{array}{l}0.70766 \\
0.07766\end{array}$ & $\begin{array}{l}-6.4 \\
-6.4\end{array}$ & & & & & & \\
\hline & Toiyabe Range & Johnson Canyon Dike & 97TY-001 & & & 9.12 & & & & $39^{\circ} 26^{6}$ & $11^{\circ} 05^{\prime}$ & & & & & & & & & \\
\hline \multirow[t]{3}{*}{$37 \mathrm{c}$} & Toiyabe Range & Aiken Creek pluton & 97тY-019 & Cretaceous & & $8.01 \pm 0.02$ & $3.9^{\mathrm{b}}$ & & & $39^{\circ} 08^{\prime} 14^{\prime \prime}$ & $117^{\circ} 07^{\prime 22}$ & & 0.70578 & -2.8 & 68.3 & 15.6 & 3.5 & 3.5 & 3.1 & 1.01 \\
\hline & & Aiken Creek pluton & 97TY-020 & Cretaceous & & $7.94 \pm 0.13$ & & & & $39^{\circ} 08$ & $117^{\circ} 07^{\prime}$ & & 0.70578 & -2.8 & 68.3 & 15.6 & 3.5 & 3.5 & 3.1 & 1.01 \\
\hline & & Aiken Creek pluton & 97TY-021 & Cretaceous & & $8.91 \pm 0.01$ & & & & $39^{\circ} 0704^{\prime \prime}$ & 117009'38" & & 0.70578 & -2.8 & 68.3 & 15.6 & 3.5 & 3.5 & 3.1 & 1.01 \\
\hline \multirow{2}{*}{ 370 } & $\begin{array}{l}\text { Tolyabe Range } \\
\text { Tivaze Range }\end{array}$ & $\begin{array}{l}\text { McLeod pluton } \\
\text { Timber }\end{array}$ & $977 Y-022$ & Cretaceous & & 6.78 & & & & $39^{\circ} 0225^{\prime \prime}$ & 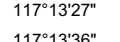 & & 070535 & -2 & & & & & & \\
\hline & & $\begin{array}{l}\text { Timbinin Creee pluton } \\
\text { Timblin Creek pluton }\end{array}$ & $\begin{array}{l}97 T Y-023 \\
97 T Y-224\end{array}$ & $\begin{array}{l}\text { Cretaceous, } 92 \mathrm{Ma} \\
\text { Cretaceous, } 92 \mathrm{Ma}\end{array}$ & & $\begin{array}{l}7.3770 .01 \\
736 \pm 0.03\end{array}$ & & & & $38^{\circ} 5957^{\prime \prime}$ & $\begin{array}{l}17 / 77^{\circ} 36^{\prime \prime} \\
117^{\prime \prime} 136^{\prime \prime}\end{array}$ & & $\begin{array}{l}0.00355 \\
0.70535\end{array}$ & $\begin{array}{l}-2 \\
-2\end{array}$ & & & & & & \\
\hline \multirow[t]{5}{*}{$37 \mathrm{c}$} & Toiyabe Range & Ophir Canyon pluton & $977 \mathrm{~T}-025$ & Cretaceous $\sim 96 \mathrm{Ma}$ & & $7.93+0.03$ & & & & 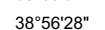 & $117^{\circ} 11^{4} 4^{11}$ & & 0.705 & -1.5 & & & & & & \\
\hline & & Ophir Canyon pluton & $977 \mathrm{~T}-026$ & Cretaceous $\sim 96 \mathrm{Ma}$ & & $7.89 \pm 0.02$ & & & & $38^{\circ} 55^{6} 40^{*}$ & $117^{\circ} 1550^{\prime \prime}$ & & 0.705 & -1.5 & & & & & & \\
\hline & & Ophir Canyon pluton & $977 \mathrm{Y}-027$ & Cretaceous $-96 \mathrm{Ma}$ & & $7.70 \pm 0.01$ & & & & $38^{\circ} 55^{6} 34^{\prime \prime}$ & $117^{\circ} 1530^{\prime \prime}$ & & 0.705 & -1.5 & & & & & & \\
\hline & & Ophir Canyon pluton & $977 \mathrm{~T}-228$ & Cretaceous $\sim 96 \mathrm{Ma}$ & & $7.84 \pm 0.02$ & & & & $38^{\circ} 5^{6} 36^{\prime \prime}$ & $117^{\circ} 1^{5} 06^{\prime \prime}$ & & 0.705 & -1.5 & & & & & & \\
\hline & & Ophir Canyon pluton & 97TY-029 & Cretaceous $\sim 96 \mathrm{Ma}$ & & $7.73 \pm 0.00$ & & & & & $117^{\circ} 5^{5}{ }^{\circ} 6^{\prime \prime}$ & & 0.705 & -1.5 & & & & & & \\
\hline \multirow{8}{*}{38} & Stillwater Range & IXL Canyon pluton & 96BR-101 & Tertiary, 29-28 Ma & & $5.74 \pm 0.04$ & $-0.2,7.0^{\mathrm{a}}$ & & $6.43-7.48$ & & $118^{\circ} 10^{\circ}$ & & 0.7051 & -0.2 & 64 & 15.2 & 4 & 3.6 & 3.8 & 0.88 \\
\hline & & IXL Canyon pluton & 966R-105 & Tertiary, 29-28 Ma & & 5.82 & & & & & $11^{\circ} 10^{\prime}$ & & 0.7051 & -0.2 & 64 & 15.2 & 4 & 3.6 & 3.8 & 0.88 \\
\hline & & IXL Canyon pluton & 966R-108 & Tertiary, 29-28 Ma & & $4.94 \pm 0.02$ & & & & & $11^{\circ} 10^{\prime}$ & $2042 \mathrm{~m}$ & 0.7051 & -0.2 & 64 & 15.2 & 4 & 3.6 & 3.8 & 0.88 \\
\hline & & IXL Canyon pluton & 968R-109 & Tertiary, 29-28 Ma & & 5.60 & & & & & $118^{\circ} 10^{\prime}$ & $1951 \mathrm{~m}$ & 0.7051 & -0.2 & 64 & 15.2 & 4 & 3.6 & 3.8 & 0.88 \\
\hline & & IXL Canyon pluton & 966R-110 & Tertiary, 29-28 Ma & & $5.16 \pm 0.01$ & & & & & $111^{\circ} 10^{\circ}$ & $1829 \mathrm{~m}$ & 0.7051 & -0.2 & 64 & 15.2 & 4 & 3.6 & 3.8 & 0.88 \\
\hline & & IXL Canyon pluton & 966R-112 & Tertiary, 29-28 Ma & & 5.80 & & & & & $118^{\circ} 10^{\circ}$ & $1707 \mathrm{~m}$ & 0.7051 & -0.2 & 64 & 15.2 & 4 & 3.6 & 3.8 & 0.88 \\
\hline & & IXL Canyon pluton & $99 G B-124$ & Tertiary, 29-28 Ma & 5.68 & & & & & $39^{\circ} 3753^{\prime \prime}$ & $118^{\circ} 10^{\prime} 41^{\prime \prime}$ & & 0.7051 & -0.2 & 64 & 15.2 & 4 & 3.6 & 3.8 & 0.88 \\
\hline & & IXL Canyon pluton & 96BR-114 & Tertiary, 29-28 Ma & & $5.955 \pm 0.02$ & & & & & $118^{\circ} 10^{\circ}$ & $1378 \mathrm{~m}$ & 0.7051 & -0.2 & 64 & 15.2 & 4 & 3.6 & 3.8 & 0.88 \\
\hline 38 & Stillwater Range & lower West Job Canyon Tuff & 96BR-119 & Tertiary, 29-28 Ma & & $5.52 \pm 0.03$ & & & & & $118^{\circ} 20^{\circ}$ & & & & & & & & & \\
\hline \multirow{7}{*}{$\begin{array}{l}38 \\
39\end{array}$} & Stillwater Range & Job Canyon Pass Tuff & 96BR-107 & Tertiary, 29-28 Ma & & 6.5550 .11 & & & & & $118^{\circ} 20^{\circ}$ & & & & & & & & & \\
\hline & Sand Springs Range & Red Top Canyon & 96BR-115 & Cretaceous, $76 \mathrm{Ma}$ & & $6.49 \pm 0.01$ & & & & & $118^{\circ} 20^{\prime}$ & & 0.7047 & 0.9 & & & & & & \\
\hline & & & 996B-116 & Cretaceous, $81 \mathrm{Ma}$ & $9.80 \pm 0.15$ & & $7.2^{\mathrm{a}}$ & & & $39^{\circ} 09^{2} 25^{\prime \prime}$ & $118^{\circ} 20^{\circ} 07^{\prime \prime}$ & & 0.7047 & 0.9 & 70.7 & 14.8 & 2.6 & 4.9 & 2.8 & 0.93 \\
\hline & & & 996B-117 & Cretaceous, $81 \mathrm{Ma}$ & $9.95 \pm 0.33$ & & & & & $39^{\circ} 09^{2} 5^{\prime \prime}$ & $118^{\circ} 20^{\circ} 07^{\prime \prime}$ & & 0.7047 & 0.9 & 70.7 & 14.8 & 2.6 & 4.9 & 2.8 & 0.93 \\
\hline & & & 996B-118 & Cretaceous, $81 \mathrm{Ma}$ & $9.68 \pm 0.01$ & & & & & $39^{\circ} 09^{2} 5^{\prime \prime}$ & $118^{\circ} 20^{\circ} 77^{\prime \prime}$ & & 0.7047 & 0.9 & 70.7 & 14.8 & 2.6 & 4.9 & 2.8 & .93 \\
\hline & & & 996B-119 & Cretaceous, $81 \mathrm{Ma}$ & $9.93 \pm 0.02$ & & & & & $39^{\circ} 09^{2} 5^{\prime \prime}$ & $118^{\circ} 20^{\circ} 077^{\prime \prime}$ & & 0.7047 & 0.9 & 70.7 & 14.8 & 2.6 & 4.9 & 2.8 & 93 \\
\hline & & & 99GB-120 & Cretaceous, $81 \mathrm{Ma}$ & $8.78 \pm 0.07$ & & & & & $39^{9} 1158^{\prime \prime}$ & $118^{\circ} 20^{\prime} 26^{\prime \prime}$ & & 0.7047 & 0.9 & 70.7 & 14.8 & 2.6 & 4.9 & 2.8 & 0.93 \\
\hline
\end{tabular}


Data Repository item 2004046

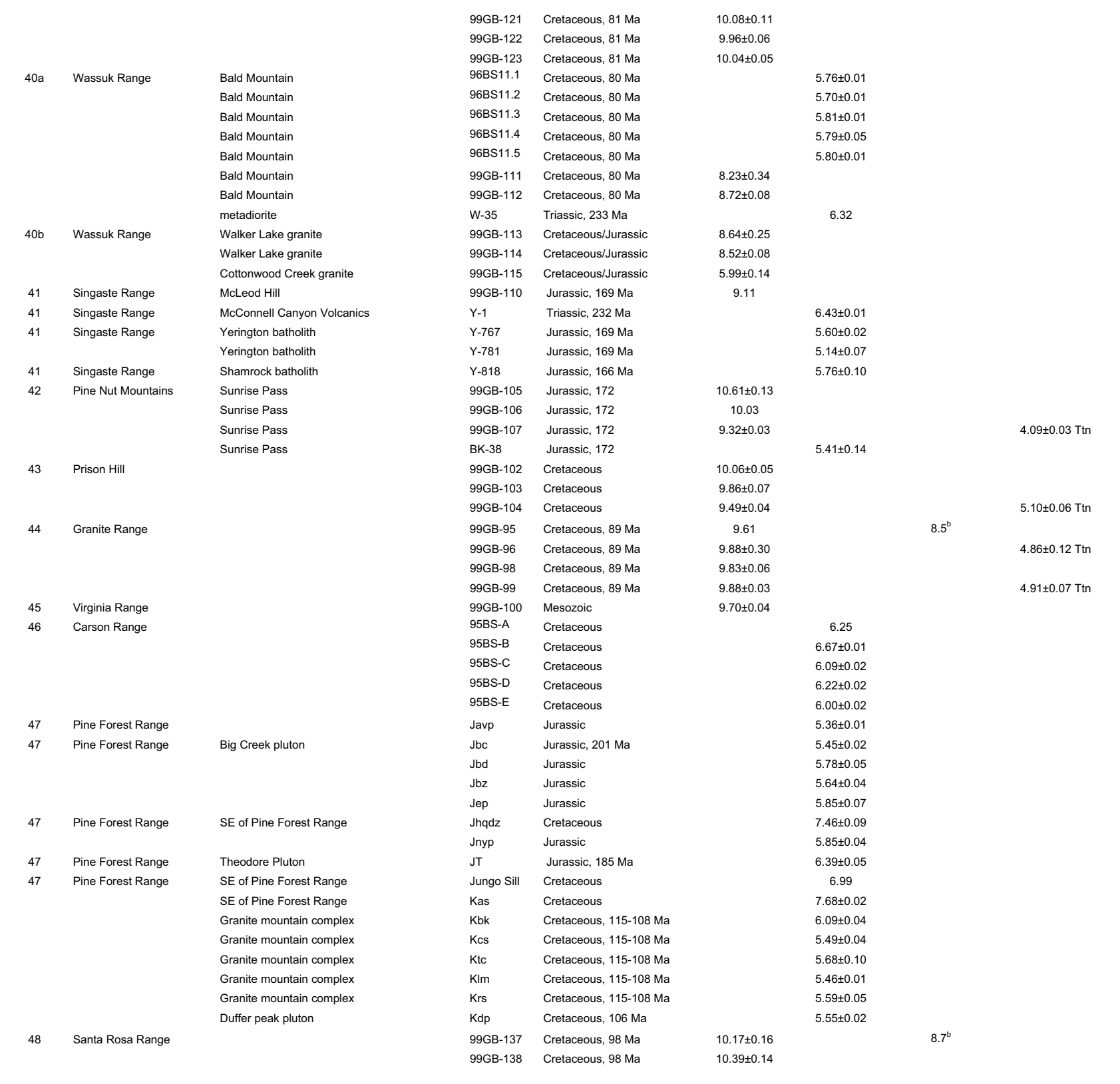

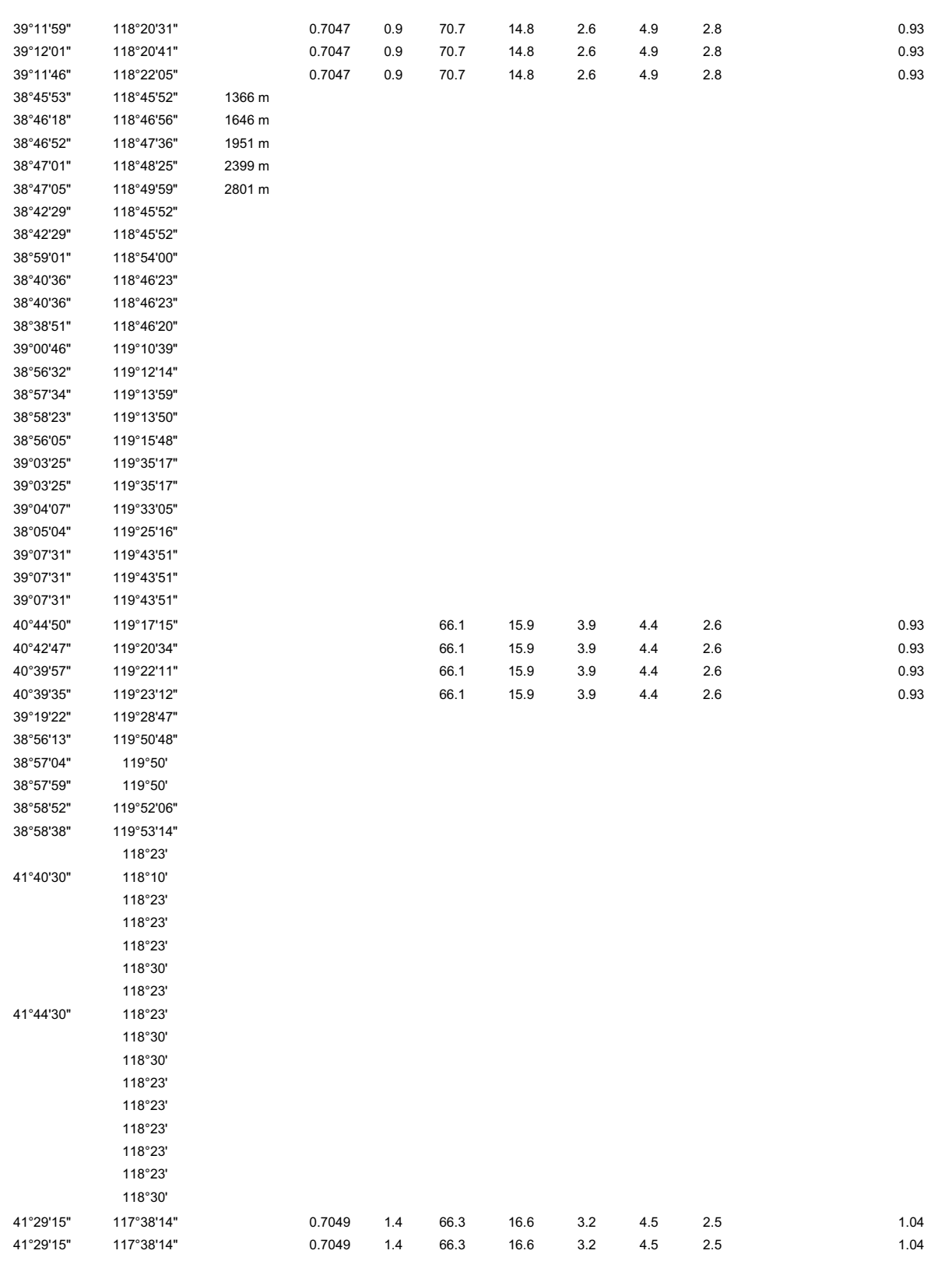


Data Repository item 2004046

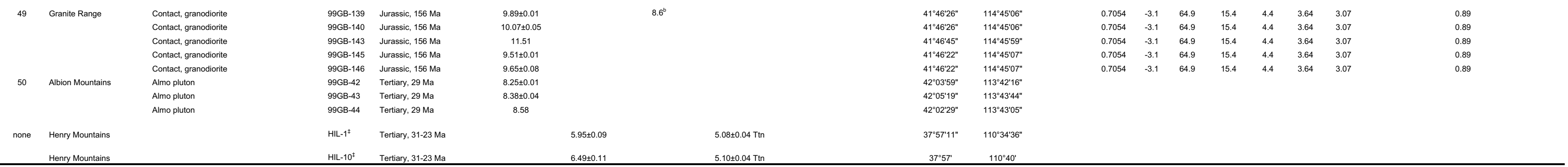

t: Map \# referss to Figure 2

†: see King et al., 2001

S: a: Solomon, 1989; b: Lee et al, 1981; c: Lee et al, 1982 


\begin{tabular}{|c|c|c|c|c|}
\hline Map \# & Mountain Range & Specific Location & Sample & References, Comments \\
\hline 1 & Wasatch Range & Little Cottonwood stock & & Crittenden et al., 1973; Farmer and DePaolo, 1983 \\
\hline 2 & Silver City Stock & & & Laughlin et al., 1969; Lee, 1984; Lee et al., 1981 \\
\hline 3,4 & Sheeprock Mountains & & & Christiansen et al., 1988 \\
\hline 5 & Desert Mountain & & & Armstrong, 1970; Lee et al., 1981; Lee, 1984 \\
\hline 6 & House Range & Notch Peak & & $\begin{array}{l}\text { Lee et al., 1986; Wright and Wooden, 1991; } \\
\text { Lee et al., 1981; Lee, } 1984\end{array}$ \\
\hline 7 & Raft River Mountains & Clear Creek Canyon & & Compton et al., 1977 \\
\hline 8 & Grouse Creek Mountains & Imigrant Pass & & $\begin{array}{l}\text { Compton et al., 1977; Wright and Wooden, 1991; } \\
\text { Lee et al., 1981; Lee, } 1984\end{array}$ \\
\hline 8 & Grouse Creek Mountains & Ingham Pass & & Compton et al., 1977; Wright and Wooden, 1991 \\
\hline 9 & Pilot Range & McGinty monzogranite & & $\begin{array}{l}\text { Hoggatt and Miller, 1981; Wright and Wooden, 1991; } \\
\text { Lee et al., 1981; Lee, } 1984\end{array}$ \\
\hline 9 & Pilot Range & Pilot Peak granitic dike & & Hoggatt and Miller, 1981 \\
\hline 10 & Silver Island Range & Crater Island South Stock & & Miller and Hoisch, 1995 \\
\hline 11 & Gold Hills & southern stock & & Stacey and Zartman, 1978; Farmer and DePaolo, 1983 \\
\hline 12 & Deep Creek Range & Ipabah stock & & $\begin{array}{l}\text { Miller et al., 1988; Wright and Wooden, 1991; } \\
\text { Lee et al., 1981; Lee, } 1984\end{array}$ \\
\hline 13 & Beaver Dam Mountains & & & Wasserburg and Lanphere, 1965, \\
\hline 14 & Kern Mountains & Tungstonia granite & & $\begin{array}{l}\text { Lee et al., 1986; Miller et al., 1988; } \\
\text { Farmer and DePaolo, 1983; Wright and Wooden, } 1991\end{array}$ \\
\hline $15 a$ & Southern Snake Range & Lexington Creek & & $\begin{array}{l}\text { Lee et al., 1970; Lee et al., 1981; } \\
\text { Lee and Chirstiansen, 1983; Lee et al., 1986; } \\
\text { Miller et al., 1988; Wright and Wooden, } 1991\end{array}$ \\
\hline $15 b$ & Southern Snake Range & Snake Creek Canyon & & $\begin{array}{l}\text { Lee et al., 1970; Lee and Christiansen, 1983; } \\
\text { Miller et al., } 1988\end{array}$ \\
\hline $15 c$ & Southern Snake Range & $\begin{array}{l}\text { Strawberry Creek/Osceola } \\
\text { granodiorite }\end{array}$ & & $\begin{array}{l}\text { Lee and Christainsen, 1983; Lee et al., 1986; } \\
\text { Miller et al., } 1988\end{array}$ \\
\hline 16 & Goshute Mountains & White Horse granite & & $\begin{array}{l}\text { Lee et al., 1981; Lee, 1984; Coats, 1987; } \\
\text { Marvin et al., 1989; Miller and Hoisch, } 1995\end{array}$ \\
\hline $17 a$ & Toano Range & Silver Zone Pass & & $\begin{array}{l}\text { Coats et al., 1965; Farmer and DePaolo, 1983; } \\
\text { Miller and Hoisch, } 1995\end{array}$ \\
\hline $17 \mathrm{~b}$ & Toano Range & Toana Springs granite & & $\begin{array}{l}\text { Lee et al., 1981; Miller et al., } 1990 \\
\text { Wright and Wooden, } 1991\end{array}$ \\
\hline 18 & Dolly Varden Mountains & Melrose pluton & & $\begin{array}{l}\text { Lee et al., 1981; Lee, 1984; } \\
\text { Zamudio et al., 1995; Miller and Hoisch, } 1995\end{array}$ \\
\hline 19 & Mormon Mountains & & & Wasserburg and Lanphere, 1965 \\
\hline 20 & Antelope Range & Kingsley granite & & Armstrong, 1970 \\
\hline 22 & Delcer Buttes & & & Coats, 1987 \\
\hline 23 & Cherry Creek Mountains & Cherry Creek granite & & Armstrong, 1963; Farmer and DePaolo, 1983 \\
\hline 24 & Egan Range & Gold Creek granite & & Armstrong, 1963 \\
\hline 24 & Egan Range & Warm Springs granite & & Armstrong, 1963 \\
\hline $25 a$ & Ruby Mountains & Little Bald Mountain & & Hose and Blake, 1976 \\
\hline $25 b$ & Ruby Mountains & Harrison Pass & & Farmer and DePaolo, 1983; Wright and Snoke, 1993 \\
\hline $25 b$ & Ruby Mountains & McCutcheon Creek & & Kistler et al., 1981; Farmer and DePaolo, 1983 \\
\hline $25 c$ & Ruby Mountains & Lamoille Canyon & RM-15 & Wright and Snoke, 1993 \\
\hline 26 & East Humboldt Range & Rattlesnake Canyon & $\mathrm{RM}-13$ & Wright and Snoke, 1993 \\
\hline 26 & East Humboldt Range & & $\mathrm{RM}-18, \mathrm{RM}-19$ & Wright and Snoke, 1993 \\
\hline 26 & East Humboldt Range & & RM-20 & MacCready et al., 1997 \\
\hline 26 & East Humboldt Range & & RM-24 & MacCready et al., 1997 \\
\hline 26 & East Humboldt Range & Angel Lake orthogneiss & RM-5 & Wright and Wooden, 1991; Wright and Snoke, 1993 \\
\hline 26 & East Humboldt Range & Horse Creek & $\mathrm{RM}-7$ & Wright and Snoke, 1993 \\
\hline 27 & Cortez Mountains & Cottonwood Canyon & & Muffler, 1964; Lee et al., 1981; Lee, 1984 \\
\hline 28 & Dry Hills & & & Muffler, 1964 \\
\hline
\end{tabular}


Troy granite lower Willow Creek

Austin pluton

Birch Creek pluton

Aiken Creek pluton

McLeod pluton

Timblin Creek pluton

Ophir Canyon pluton

IXL Canyon pluton

Job Canyon Pass Tuff

Red Top Canyon

\section{Bald Mountain}

metadiorite

Walker Lake granite

Cottonwood Creek granite

McLeod Hill

Mcconnell Canyon volcanics

Yerington batholith

Shamrock batholith

Sunrise Pass

Big Creek pluton

SE of Pine Forest Range

Theodore Pluton

SE of Pine Forest Range

SE of Pine Forest Range

Granite mountain complex

Granite mountain complex

Duffer peak pluton

Granite mountain complex

Contact, granodiorite
Lee et al., 1981; Lee, 1984;

Kleinhampl and Ziony, 1985

Lee et al., 1981; Lee, 1984; Brooks and Snee, 1996

Kleinhampl and Ziony, 1985

Silberman and McKee, 1971; Elison et al.,1990;

Farmer and DePaolo, 1983

Erickson et al., 1978; Lee et al., 1981; Lee, 1984

Silberman et al., 1974; Farmer and DePaolo, 1983;

Elison et al., 1990

Conrad and McKee, 1995

Johnson, 1977; Lee et al., 1981; Lee, 1984

Krueger and Schilling, 1971; Lee et al., 1981; Lee, 1984; Farmer and DePaolo, 1983; Elison et al., 1990

McKee, 1976, Wright and Wooden, 1991

Lee et al., 1981; Lee, 1984;

Kleinhampl and Ziony, 1985; Wright and Wooden, 1991

Kleinhampl and Ziony, 1985

Smith and Wright, 1988; Wright and Wooden, 1991

Smith and Wright, 1988; Wright and Wooden, 1991

Lee et al., 1981; Farmer and DePaolo, 1983; Lee, 1984; Elison et al., 1990; John, 1995;

J.E. Conrad and D.A. John, unpub. data, 1995

John, 1995; J.E. Conrad and D.A. John,

unpub. data, 1995

Willden and Speed, 1974; Lee et al., 1981;

Farmer and DePaolo, 1983; Lee, 1984;

Elison et al., 1990; John, 1992

Bingler, 1978

W-35

Dilles and Wright, 1988

Stewart et al., 1981

Stewart et al., 1981

Dilles and Wright, 1988

Dilles and Wright, 1988

$\mathrm{Y}-1$

Y-767, Y-781

Dilles and Wright, 1988

Dilles and Wright, 1988

Dilles and Wright, 1988

John, 1992

Krueger and Schilling, 1971; Lee et al., 1981; Lee, 1984

Moore, 1969

Lintz, 1993

Javp, Jbd, Jbz, Jep

$\mathrm{Jbc}$

Wright, unpublished data

Wyld, 1996

Jhqdz, Jnyp

JT

Wright, unpublished data

Wyld, 1996

Jungo Sill Wright, unpublished data

Kas

Kbk, Kcs

Wright, unpublished data

Wright, unpublished data

Kcs

$\mathrm{Kdp}$

$\mathrm{KIm}, \mathrm{Krs}, \mathrm{Ktc}$

Wright, unpublished data

Wright, unpublished data

Wright, unpublished data
Silberman and Mckee, 1971; Elison et al., 1990;

Lee et al., 1981; Farmer and DePaolo, 1983; Lee, 1984

Coats et al., 1965; Miller and Hoisch, 1995;

Lee et al., 1981; Lee, 1984

Forrest et al., 1994

Nelson, 1998 
Armstrong, R.L., 1963, Geochronology and geology of the eastern Great Basin in Nevada and Utah [PhD thesis]: New Haven, Yale University.

_ , 1970, Geochronology of Tertiary igneous rocks, eastern Basin and Range Province, western Utah, eastern Nevada, and vicinity, U.S.A.: Geochimica et

Cosmochimica Acta, v. 34, p. 203-232.

Bingler, E.C., 1978, Geologic map of the Schurz quadrangle: Nevada Bureau of Mines and Geology map 60.

Brooks, W.E., and Snee, L.W., 1996, Timing and effect of detachment-related potassium metasomatism on ${ }^{40} \mathrm{Ar} /{ }^{39} \mathrm{Ar}$ ages from the Windous Butte Formation, Grant Range, Nevada: U. S. Geological Survey Bulletin 2154, 25 p.

Christiansen, E.H., Stuckless, J.S., Funkhouser, M.M.J., and Howell, K.H., 1988, Petrogenesis of rare-metal granites from depleted crustal sources; an example from the Cenozoic of western Utah, U.S.A, in Taylor, R.P., and Strong, D.F., eds., Recent advances in the geology of granite-related mineral deposits., Volume Special Volume 39 - Canadian Institute of Mining and Metallurgy, p. 307-321.

Coats, R.R., 1987, Geology of Elko County, Nevada: Nevada Bureau of Mines and Geology Bulletin 101, 112 p., p. 112.

Coats, R.R., Marvin, R.F., and Stern, T.W., 1965, Reconnaissance of mineral ages of plutons in Elko County, Nevada, and vicinity: U. S. Geological Survey Professional Paper 525-D, p. D11-D15.

Compton, R.R., Todd, V.R., Zartman, R.E., and Naeser, C.W., 1977, Oligocene and Miocene metamorphism, folding, and low-angle faulting in northwestern Utah: Geological Society of America Bulletin, v. 88, p. 1237-1250.

Conrad, J.E., and McKee, E.H., 1995, High-precision ${ }^{40} \mathrm{Ar} /{ }^{39} \mathrm{Ar}$ ages of rhyolitic host rock and mineralized veins at the Sleeper Deposit, Humboldt County, Nevada, in Coyner, A.R., and Fahey, P., eds., Geology and ore deposits of the American Cordillera; symposium proceedings.: Reno, NV, United States, Geological Society of Nevada, p. 257-262.

Crittenden, M.D., Jr., Stuckless, J.S., Kistler, R.W., and Stern, T.W., 1973, Radiometric dating of intrusive rocks in the Cottonwood area, Utah: Journal of Research of the U. S. Geological Survey, v. 1, p. 173-178.

Dilles, J.H., and Wright, J.E., 1988, The chronology of early Mesozoic arc magmatism in the Yerington District of western Nevada and its regional implications:

Geological Society of America Bulletin, v. 100, p. 644-652.

Elison, M.W., Speed, R.C., and Kistler, R.W., 1990, Geologic and isotopic constraints on the crustal structure of the northern Great Basin: Geological Society of America Bulletin, v. 102, p. 1077-1092.

Erickson, R.L., Silberman, M.L., and Marsh, S.P., 1978, Age and composition of igneous rocks, Edna Mountain Quadrangle, Humboldt County, Nevada: Journal of Research of the U. S. Geological Survey, v. 6, p. 727-743.

Farmer, G.J., and DePaolo, D.J., 1983, Origin of Mesozoic and Tertiary granite in the Western United States and implications for pre-Mesozoic crustal structure 1. Nd and $\mathrm{Sr}$ isotopic studies in the geocline of the northern Great Basin: Journal of Geophysical Research, v. 88, p. 3379-3401. 
Forrest, S.E., Miller, E.L., and Wright, J.E., 1994, Oligocene plutonism and associated crustal thinning in the southern Albion Mountains, Idaho, Geological Society of America Abstracts with Programs, Volume 26, p. 192.

Hoggatt, W.C., and Miller, D.M., 1981, K-Ar ages of intrusive rocks of the Pilot Range, Nevada and Utah: Isochron/West, v. 30, p. 21-22.

Hose, R., and Blake, M., 1976, Geology and Mneral Resources of White Pine County, Nevada: Nevada Bureau of Mines and Geology Bulletin 85, p. 1-35.

John, D.A., 1992, Late Cenozoic volcanotectonic evolution of the southern Stillwater Range, west-central Nevada, in Craig, S., ed., Geological Society of Nevada; proceedings volume; Walker Lane symposium; Structure, tectonics \& mineralization of the Walker Lane., p. 64-92.

John, D.A., and Pickthorn, W.J., 1995, Alteration and stable isotope studies of a deep meteoric-hydrothermal system in the Job Canyon Caldera and IXL Pluton, southern Stillwater Range, Nevada, in Coyner, A.R., and Fahey, P.L., eds., Geology and ore deposits of the American Cordillera; symposium proceedings.: Reno, NV, United States, Geological Society of Nevada, p. 733-756.

Johnson, M.G., 1977, Geology and mineral deposits of Pershing County, Nevada: Nevada Bureau of Mines and Geology Bulletin, v. 89, $121 \mathrm{p}$.

King, E.M., Valley, J.W., Davis, D.W., and Kowallis, B.J., 2001, Empirical determination of oxygen isotope fractionation factors for titanite with respect to zircon and quartz: Geochimica et Cosmochimica Acta, in press.

Kistler, R.W., Ghent, E.D., and O' Neil, J.R., 1981, Petrogenesis of garnet two-mica granites in the Ruby Mountains, Nevada: Journal of Geophysical Research. B, v. 86, p. 10591-10606.

Kleinhampl, F., and Ziony, J., 1985, Geology of Northern Nye County, Nevada: Nevada Bureau of Mines and Geology Bulletin 99A, $172 \mathrm{p}$.

Krueger, H.W., and Schilling, J.H., 1971, Geochron/ Nevada Bureau of Mines K/ Ar age determinations; list 1: Isochron/West, v. 1, p. 9-14.

Laughlin, A.W., Lovering, T.S., and Mauger, R.L., 1969, Age of some Tertiary igneous rocks from the East Tintic District, Utah: Economic Geology, v. 64, p. 915-918.

Lee, D.E., 1984, Analytical data for a suite of granitoid rocks from the Basin and Range Province, $54 \mathrm{p}$.

Lee, D.E., and Christiansen, E.H., 1983, The granite problem as exposed in the southern Snake Range, Nevada: Contributions to Mineralogy and Petrology, v. 83, p. 99116.

Lee, D.E., Friedman, I., and Gleason, J.D., 1981, Map showing the oxygen isotope composition of granitoid rocks of the Basin-Range Province: U. S. Geological Survey Miscellaneous Field Studies Map 1305, 1 sheet.

- 1986, The oxygen isotope composition of selected quartzites of White Pine County, Nevada, and nearby areas: Reston, VA, United States, U. S. Geological Survey, 6 p.

Lee, D.E., Friedmann, I., and Gleason, J.D., 1982, The oxygen isotope composition of granitoid and sedimentary rocks of the southern Snake Range, Nevada: Contributions to Mineralogy and Petrology, v. 79, p. 150-158.

Lee, D.E., Marvin, R.F., Stern, T.W., and Peterman, Z.E., 1970, Modification of potassium-argon ages by Tertiary thrusting in the Snake Range, White Pine 
County, Nevada, U. S. Geological Survey Professional Paper 700-D, p. D92D102.

Lintz, J., 1993, Lake Tahoe field trip, in Lahren, M.M., Trexler, J.H., and Spinosa, C., eds., Crustal evolution of the Great Basin and the Sierra Nevada., p. 263-276.

MacCready, T., Snoke, A.W., Wright, J.E., and Howard, K.A., 1997, Mid-crustal flow during Tertiary extension in the Ruby Mountains core complex, Nevada:

Geological Society of America Bulletin, v. 109, p. 1576-1594.

McKee, E.H., 1976, Geologic map of the Austin Quadrangle, Lander County, Nevada: United States Geological Survey map GQ-1307.

Miller, D.M., and Hoisch, T.D., 1995, Jurassic tectonics of northeastern Nevada and northwestern Utah from the perspective of barometric studies, in Miller, D.M., and Busby, C., eds., Jurassic magmatism and tectonics of the North American Cordillera., Volume 299: Special Paper - Geological Society of America:

Boulder, CO, United States, Geological Society of America (GSA), p. 267-294.

Miller, D.M., Nakata, J.K., and Glick, L.L., 1990, K-Ar ages of Jurassic to Tertiary plutonic and metamorphic rocks, northwestern Utah and northeastern Nevada: U. S. Geological Survey Bulletin 1906, 18 p.

Miller, E.L., Gans, P.B., Wright, J.E., and Sutter, J.F., 1988, Metamorphic history of the east-central Basin and Range Province; tectonic setting and relationship to magmatism, in Ernst, W.G., ed., Metamorphism and crustal evolution of the Western United States: Rubey Volume 7, p. 649-682.

Moore, J.G., 1969, Geology and mineral deposits of Lyon, Douglas, and Ormsby Counties, Nevada: Nevada Bureau of Mines and Geology Bulletin, v. 75, 45 p.

Muffler, L.J.P., 1964, Geology of the Frenchie Creek Quadrangle North-Central Nevada: U.S. Geological Survey Bulletin 1179, 99 p.

Nelson, S.T., 1998, Reevaluation of the central Colorado Plateau laccoliths in the light of new age determinations, in Friedman, J.D., and Huffman, C., eds., Laccolith complexes of southeastern Utah; time of emplacement and tectonic setting; workshop proceedings: U. S. Geological Survey Bulletin 2158, p. 37-39.

Silberman, M.L., Berger, B.R., and Koski, R.A., 1974, K-Ar Age Relations of Granodiorite Emplacement and Tungsten and Gold Mineralization near the Getchell Mine, Humboldt County, Nevada: Economic Geology, v. 69, p. 646-656.

Silberman, M.L., and McKee, E.H., 1971, K-Ar ages of granitic plutons in north-central Nevada: Isochron/West, v. 1, p. 15-32.

Smith, D.L., and Wright, J.E., 1988, Late Cretaceous plutonism and associated deformation and metamorphism in central Nevada: Geological Society of America Abstracts with Programs, v. 20, p. 17-18.

Solomon, G.C., 1989, An ${ }^{18} \mathrm{O} /{ }^{16} \mathrm{O}$ study of Mesozoic and early Tertiary granitic batholiths of the southwestern North American Cordillera [PhD thesis], California Institute of Technology.

Stacey, J.S., and Zartman, R.E., 1978, A lead and strontium isotopic study of igneous rocks and ores, from the Gold Hill mining district, Utah: Utah Geology, v. 5, p. 115.

Stewart, J., Reynolds, M., and Johannesen, D., 1981, Geologic map fo the Mount Grant quadrangle, Lyon and Mineral counties, Nevada: U.S. Geological Survey Miscellaneous Field Studies Map MF-1278. 
Wasserburg, G.J., and Lanphere, M.A., 1965, Age determinations in the Precambrian of Arizona and Nevada: Geological Society of America Bulletin, v. 76, p. 735-758.

Willden, R., and Speed, R.C., 1974, Geology and mineral deposits of Churchill County, Nevada: Nevada Bureau of Mines and Geology Bulletin 83, 95 p.

Wright, J.E., and Snoke, A.W., 1993, Tertiary magmatism and mylonitization in the Ruby-East Humboldt metamorphic core complex, northeastern Nevada; U-Pb geochronology and $\mathrm{Sr}, \mathrm{Nd}$, and $\mathrm{Pb}$ isotope geochemistry: Geological Society of America Bulletin, v. 105, p. 935-952.

Wright, J.E., and Wooden, J.L., 1991, New Sr, Nd, and $\mathrm{Pb}$ isotopic data from plutons in the northern Great Basin; implications for crustal structure and granite petrogenesis in the hinterland of the Sevier thrust belt: Geology, v. 19, p. 457-460.

Wyld, S.J., 1996, Early Jurassic deformation in the Pine Forest Range, Northwest Nevada, and implications for Cordilleran tectonics: Tectonics, v. 15, p. 566-583.

Zamudio, J.A., and Atkinson, W.W., Jr., 1995, Mesozoic structures of the Dolly Varden Mountains and Currie Hills, Elko County, Nevada, in Miller, D.M., and Busby, C., eds., Jurassic magmatism and tectonics of the North American Cordillera: Geological Society of America Special Paper 299, p. 295-311. 